\section{Keeping neurons on track}

During development, the chemokine SDF1 guides migrating neurons to different targets. However, many distinct sources of SDF1 exist and it is unclear how neurons choose the correct one. Knaut and colleagues have found that zebrafish trigeminal sensory neurons follow a dynamic track of SDF1, which depends on a shift in the SDF1 expression domain towards the final destination and on the clearance of SDF1 transcript and protein at the back of the track (J. Cell Biol. 200, 337-355; 2013).

The authors first observed that these particular neurons follow a narrow band of SDF1 expression that shifts forwards in the direction of their migration. They found that switching on SDF1 expression at the front of the band guides the movement of neurons, whereas two mechanisms ensure the rapid clearance of SDF1 at the back. The SDF1 mRNA band is broadened in embryos lacking the miRNAprocessing enzyme DICER, resulting in guidance defects. The authors show that miR-430 targets SDF1 for degradation to ensure clearance of SDF1 transcripts. Furthermore, cells surrounding the migrating neurons express a particular SDF1 receptor, CXCR7b, which acts as a sink for the SDF1 protein, therefore reducing SDF1 action on the CXCR4b receptors on migrating neurons. How the passage of the neurons triggers a shift in SDF1 expression at the front of the migrating cells and miR-430- and CXCR7b-mediated clearance of SDF1 at the back are questions for future studies.

NLB

\section{RIF1 mediates repair pathway decisions}

Non-homologous end joining (NHEJ) is particularly important for repair of double-strand breaks (DSBs) in the G1 stage of the cell cycle, when there is no homologous chromosome to act as a template for repair by homologous recombination. How the correct repair pathway is set up is unclear, but previous work suggested opposing roles for the homologous recombination factor BRCA1 and the NHEJ protein 53BP1 in end resection, an early step in homologous recombination. Several recent studies have now shed light on this mechanism.

The groups of de Lange (Science 339, 700-704; 2013), Nussenzweig (Science 339, 711-715; 2013), Durocher (Mol. Cell http:// doi.org/kgk; 2013) and Boulton (Mol. Cell http://doi.org/kgm; 2013) all identify RIF1 as an effector of 53BP1 that is recruited to DSBs in G1 to prevent end resection. In the absence of RIF1, DSBs are hyperresected and cells show G1-specific hypersensitivity to ionizing radiation. The Boulton and Nussenzweig groups find that RIF1-null mice are deficient in class switch recombination, demonstrating a critical role for RIF1 in NHEJ in vivo. The Durocher, de Lange and Boulton groups further elucidate how RIF1 influences repair pathway choice, and find that RIF1 together with 53BP1 blocks BRCA1 recruitment in G1. Conversely, BRCA1 prevents the accumulation of RIF1 to DSBs in S or G2, and Durocher and colleagues find that the BRCA1 cofactor CtIP, phosphorylated by

\title{
Regulating nutrient-stress-induced autophagy
}

Autophagy maintains cellular homeostasis in response to various stresses, including nutrient starvation. Vps34, a PtdIns-3-OH kinase, induces autophagy but also has non-autophagyrelated functions. How Vps34 can exert these different roles has been unclear.

Guan and colleagues (Cell 152, 290-303; 2013) studied the regulation of Vps34 following glucose starvation by isolating four different Vps34 sub-groups, which were divided into nonautophagy and pro-autophagy complexes. They show that following energy deprivation, the known nutrient sensor AMPK (AMP-activated protein kinase) regulates the activity of Vps34 complexes either by inhibiting non-autophagy complexes or by activating pro-autophagy complexes. By phosphorylating Vps34 in the non-autophagy complex, AMPK suppresses overall PtdIns(3)P production, which protects the cells from starvation. In contrast, during starvation, AMPK phosphorylates Beclin-1 in the pro-autophagy complex (which also contains the Beclin-1-binding protein ATG14L) to induce autophagy. Interestingly, ATG14L suppresses AMPK-mediated phosphorylation of Vps34 in this complex, resulting in an increased level of the autophagy-related pool of PtdIns(3)P. This indicates that distinct Vps34 complexes are regulating the level of PtdIns(3)P in different cellular compartments. Thus, through differential regulation of Vps34 complexes, AMPK can optimize the cellular response to nutrient stress.

CDK1 (cyclin-dependent kinase 1), cooperates with BRCA1 in RIF1 exclusion in S/G2. Thus, mutual antagonism between 53BP1-RIF1 and BRCA1-CtIP ensures correct repair pathway choice.

CKR

\section{Intestinal tumour initiation by dedifferentiation}

Intestinal tumorigenesis often involves Wnt pathway activation and subsequent transformation and expansion of the intestinal crypt stem cell compartment. Greten and colleagues now reveal that this process may also involve dedifferentiation of non-stem intestinal epithelial cells into tumour-initiating cells (Cell 152, 25-38; 2013).

The authors observed that a genetic mouse model harbouring constitutively active $\beta$-catenin in intestinal epithelial cells (IECs) exhibited expansion of intestinal crypt stem cells, reduction of differentiated enterocytes and NF- $\kappa B$ pathway activation in IECs. Concomitant genetic inhibition of NF- $\kappa \mathrm{B}$ signalling increased mouse survival by reducing crypt stem cell expansion, downregulating Wnt-pathway-dependent target genes associated with intestinal stem cells and promoting expression of genes associated with a more differentiated phenotype. These effects were attributable to the direct interaction of NF- $\kappa \mathrm{B}$ with $\beta$-catenin to regulate gene transcription. Combined genetic activation of the Wnt and NF- $\kappa$ B pathways increased proliferative crypt stem cell numbers, and also led to the formation of crypt-like foci outside the villus epithelium, suggesting that these originated from non-stem villus cells that acquired stem cell properties. Using ex vivo organoid culture and in vivo mouse genetic-lineage tracing experiments, the authors demonstrated that non-stem IECs could indeed dedifferentiate and obtain tumour-initiating properties following hyperactivation of $\beta$-catenin. These findings demonstrate that conversion between non-stem and stem-like cell types can promote tumour initiation and highlight the importance of cell-type plasticity in cancer development.

AIZ

By Nathalie Le Bot, Christina Karlsson Rosenthal, Maria Trajkovska and Alexia-Ileana Zaromytidou 